\title{
MicroRNA-132 promotes estradiol synthesis in ovarian granulosa cells via translational repression of Nurr1
}

Shaogen $\mathrm{Wu}^{1+}$, Haixiang Sun ${ }^{1 \dagger}$, Qun Zhang ${ }^{1}$, Yue Jiang ${ }^{1}$, Ting Fang ${ }^{1}$, Isabelle Cui ${ }^{2}$, Guijun Yan ${ }^{1 *}$ and Yali Hu ${ }^{1 *}$

\begin{abstract}
Background: Estrogen synthesis is an important function of the mammalian ovary. Estrogen plays important roles in many biological processes, including follicular development, oocyte maturation and endometrial proliferation, and dysfunctions in estrogen synthesis contribute to the development of polycystic ovary syndrome and premature ovarian failure. Classical signaling cascades triggered by follicle-stimulating hormone induce estrogen synthesis via the upregulation of Cyp19a1 in granulosa cells (GCS). This study aimed to determine the effect of microRNA-132 (miR-132) on estradiol synthesis in GCs.
\end{abstract}

Methods: Primary mouse GCs were collected from ovaries of 21-day-old immature ICR mice through follicle puncture. GCs were cultured and treated with the stable cyclic adenosine monophosphate analog 8-Br-cAMP or transfected with miR-132 mimics, Nurr1-specific small interfering RNA oligonucleotides and Flag-Nurr1 plasmids. Concentrations of estradiol and progesterone in culture medium were determined by an automated chemiluminescence-based assay. Quantitative real time PCR and western blot were performed to identify the effect of miR-132 on Cyp 19a1, Cyp11a1 and an orphan nuclear receptor-Nurr1 expression in GCs. Direct suppression of Nurr1 via its 3'-untranslated region by miR-132 were further verified using luciferase reporter assays.

Results: The expression level of miR-132 in cultured mouse GCs was significantly elevated during $48 \mathrm{~h}$ of treatment with 8-Br-cAMP. The synthesis of estradiol increased after the overexpression of miR-132 in mouse GCs. The real-time PCR results demonstrated that miR-132 induced the expression of Cyp19a1 significantly. Nurr1, an orphan nuclear receptor that suppresses Cyp19a1 expression, was found to be a direct target of miR-132. Nurr1 was suppressed by miR-132, as indicated by a luciferase assay and Western blotting. The knockdown of Nurr primarily elevated the synthesis of estradiol and partially attenuated the miR-132-induced estradiol elevation, and the ectopic expression of Flag-Nurr1 abrogated the stimulatory effect of miR-132 on estradiol synthesis in mouse GCs.

Conclusions: Our findings suggest that miR-132 is involved in the CAMP signaling pathway and promotes estradiol synthesis via the translational repression of Nurr1 in ovarian GCs.

Keywords: microRNA-132, Estradiol, Granulosa cell, Nurr1, Cyp19a1

\footnotetext{
*Correspondence: yanguijun55@163.com; glyyhuyli@163.com

${ }^{\dagger}$ Equal contributors

'Reproductive Medicine Center, Department of Obstetrics and Gynecology,

Nanjing Drum Tower Hospital, Nanjing University Medical School, Nanjing,

China

Full list of author information is available at the end of the article
}

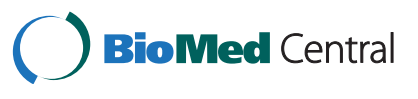

(c) 2015 Wu et al. Open Access This article is distributed under the terms of the Creative Commons Attribution 4.0 International License (http://creativecommons.org/licenses/by/4.0/), which permits unrestricted use, distribution, and reproduction in any medium, provided you give appropriate credit to the original author(s) and the source, provide a link to the Creative Commons license, and indicate if changes were made. The Creative Commons Public Domain Dedication waiver (http://creativecommons.org/publicdomain/zero/1.0/) applies to the data made available in this article, unless otherwise stated. 


\section{Background}

Ovarian steroid hormones such as estradiol $\left(E_{2}\right)$ play important roles in many biological processes, including ovarian follicular development, oocyte maturation, endometrial proliferation and mammary gland development $[1,2]$. In addition, dysfunctions in estrogen synthesis are associated with the development of polycystic ovary syndrome and premature ovarian failure [3, 4]. According to the traditional two-step theory of $E_{2}$ biosynthesis, androgen is produced from cholesterol in theca cells and converted into $\mathrm{E}_{2}$ via cytochrome $\mathrm{P} 450$ aromatase, a rate-limiting enzyme for estrogen synthesis, in granulosa cells (GCs) [5]. Follicle-stimulating hormone (FSH) is a glycoprotein hormone that is produced by the anterior pituitary gland. This gonadotropin plays an essential role in steroidogenesis of ovarian GCs. The binding of FSH to its receptor (FSHR) on the surface of GCs in immature preantral follicles activates the effector adenylyl cyclase, which leads to the synthesis and upregulation of the intracellular second messenger cyclic adenosine monophosphate (cAMP) [6]. By activating multiple signaling cascades, FSH triggers the specific, time-related expression of genes, such as Cyp19a1, and promotes the proliferation and differentiation of GCs [7]. FSH induces the phosphorylation of the cAMP response element binding protein (CREB), which transactivates Cyp19a1 by binding to a cAMP-responsive element-like sequence (CLS) in its proximal promoter (PII promoter) [8-10]. Besides classical regulations in the FSH pathway, epigenetic mechanisms remain to be elucidated, which will increase our understanding of ovarian physiology.

MicroRNAs (miRNAs) are small noncoding RNAs that are 20-24 nucleotides in length and are endogenously expressed in most eukaryotes. Previous studies demonstrated that miRNAs play important roles in diverse biological processes, such as development, inflammation and tumorigenesis [11]. The primary mechanism by which miRNAs regulate gene expression is via posttranscriptional binding to the 3'-untranslated region (3'UTR) of mRNAs, which leads to either degradation or translational repression of the mRNA. In the ovary, many miRNAs are involved in the proliferation, apoptosis, and differentiation of GCs [12, 13]. Some miRNAs have recently been reported to influence steroid hormone release from human ovarian GCs based on a genome-scale miRNA screen [14]. Studies examining miRNA-regulated $E_{2}$ biosynthesis determined that miR224 [15] and miR-383 [16] play important roles in the TGF- $\beta /$ Smads pathway by targeting Smad4 and RBMS1, respectively. The Cyp19a1 gene has also been confirmed to be a direct target of miR-378 [17] and miR-98 [18].

Among the miRNAs that are involved in the cAMP signaling pathway, miR-132 has been demonstrated to be upregulated in rat GCs by either cAMP [19] or FSH treatment [20] and in periovulatory mouse granulosa cells (mGCs) after LH/hCG induction [21]. A recent study in polycystic ovary syndrome patients showed that the expression levels of miR-132 in follicular fluid were significantly lower in patients than in controls [22]. They also found that overexpression of miR-132 increased $E_{2}$ secretion from KGN, a steroidogenic human granulosalike tumor cell line. These findings suggest that miR-132 may play diverse roles such as steroidogenesis in different developmental stage of granulose cells. The functions of miR-132 may be related to the fact that cAMP mediates divergent pathways depending on the differential status of GCs [23]. Our aims of this study are to determine if miR-132 is involved in the cAMP pathway in primary cultured $\mathrm{mGCs}$ isolated from immature mice and to investigate the role of miR-132 in $E_{2}$ synthesis using a relatively low plating density to retain the estrogenic phenotype of mGCs [24]. Our study also identified Nurr1 as a direct target of miR-132, which mediates the regulation of $E_{2}$ synthesis by miR-132 in mGCs.

\section{Methods \\ Animals}

Three-week-old ICR mice were purchased from the Lab Animal Center of Yangzhou University (Yangzhou, China). All animals were maintained in the Animal Laboratory Center of Drum Tower Hospital (Nanjing, China) on a 12-h/12-h light/dark cycle (lights off at 19:00), with food and water available ad libitum. All animal experiments were approved by the Institutional Animal Care and Use Committee at Nanjing Drum Tower Hospital (SYXK 20014-0052).

\section{Isolation and culture of primary $\mathrm{mGCs}$}

A previously described in-house method [25] was performed to isolate mGCs from the ovaries of 21-day-old immature mice. Briefly, the ovaries were harvested and separated from the surrounding fat. After the ovaries had been punctured repeatedly with 25 gauge needles, the mGCs were collected and plated in DMEM/F12 (Gibco, Life Technologies, Carlsbad, CA, USA) containing 10 \% FBS (Gibco), 1 mM sodium pyruvate (HyClone, Thermo Scientific, South Logan, UT, USA), 2 mM L-glutamine (Gibco), and $1 \%$ antibiotics $(100 \mathrm{U} / \mathrm{ml}$ penicillin and $100 \mu \mathrm{g} / \mathrm{ml}$ streptomycin; Gibco). The medium was replaced $24 \mathrm{~h}$ after plating to remove any unattached cells. The mGCs were cultured in medium at $37{ }^{\circ} \mathrm{C}$ in a humidified environment with $5 \% \mathrm{CO}_{2}$ and were used after the first passage. At $24 \mathrm{~h}$ after plating, the cells were placed in phenol red-free DMEM/F12 (HyClone) supplemented with $2 \%$ charcoal/dextran-treated fetal bovine serum (C-FBS; HyClone) for $48 \mathrm{~h}$. The cells were subsequently treated with medium alone or with $1 \mathrm{mM}$ 8-bromoadenosine 3',5'-cyclic monophosphate 
(8-Br-cAMP) (Sigma, St. Louis, MO, USA) for 0, 3, 6, 12,24 or $48 \mathrm{~h}$. Total RNA was isolated, and the expression of miR-132 was analyzed using quantitative polymerase chain reaction (PCR).

\section{Immunofluorescence staining}

mGCs were plated on $18 \mathrm{~mm}$ microcover glasses (Matsunami, Osaka, Japan) for $24 \mathrm{~h}$ and subsequently fixed with $4 \%$ paraformaldehyde in PBS for $30 \mathrm{~min}$ at room temperature. The cells were then washed with PBS and permeabilized with $0.2 \%$ Triton X-100 in PBS for $15 \mathrm{~min}$ at room temperature. After being blocked with $1 \%$ BSA in PBS, cells were stained for FSHR by incubation with a 1:100 dilution of an anti-FSHR polyclonal antibody (Bioworld Technology, St. Louis Park, MN, USA), followed by incubation with a 1:200 dilution of an Alexa Fluor 488-conjugated goat anti-rabbit IgG (Molecular Probes, Life Technologies, Carlsbad, CA, USA) for $1 \mathrm{~h}$. PBS was used as negative controls for primary and secondary antibodies to exclude nonspecific staining. Nuclei were stained with a 1:5000 dilution of DAPI (Vector Laboratories, Burlingame, CA, USA). Images were visualized using a FLUOVIEW FV10i confocal microscope system (Olympus, Tokyo, Japan).

\section{Immunohistochemistry}

Formalin-fixed paraffin-embedded 21-day-old immature mice ovaries were serially sectioned, dewaxed with $x y-$ lene and rehydrated through a graded alcohol series. Sections were then treated with $3 \%$ hydrogen peroxide to quench endogenous peroxidase activity, microwaved sequentially to retrieve antigen, and incubated in blocking solution for $1 \mathrm{~h}$. Sections were then incubated with a 1:100 dilution of an anti-FSHR polyclonal antibody (Bioworld Technology) overnight at $4{ }^{\circ} \mathrm{C}$. The next day, the sections were incubated with goat anti-rabbit secondary antibody $\mathrm{ABC}$ detect kit (ZSBio, Beijing, China) at $37^{\circ} \mathrm{C}$ for $30 \mathrm{~min}$, and then stained with 3,30-diaminobenzidine $(\mathrm{DAB})$ and counterstained with hematoxylin. Negative control sections were processed concurrently using PBS and similarly pre-treated.

\section{Plasmid construction}

NURR1 cDNA [GeneBank: NM_006186.3] was synthesized and amplified from the total RNA of human endometrial stromal cells using the SuperScript III One-Step RT-PCR System with the Platinum Taq High Fidelity Kit (Invitrogen, Life Technologies, Carlsbad, CA, USA) and the following primers: 5'-CGACACTGTCCACCTTTA ATTTC-3' and 3'-TTTAGGGATCAAGGGGGCTA-5'. A second PCR step was performed using the Platinum Pfx DNA Polymerase (Invitrogen) and the following primers: 5'-TATAAGATCTGATGCCTTGTGTTCAGGCGCAG-3' and 5'-TAGCGGTACCTTAGAAAGGTAAAGTGTCC
AG-3'. To create a Flag-Nurr1 protein expression vector, fragments harboring full-length NURR1 were cloned into pFLAG-CMV-2 (Sigma) using the BglII and KpnI restriction sites (Promega, Madison, WI, USA). The wild-type sequence of the Nurr1 3'-UTR [GeneBank: NM_013613.2] that contains the miR-132 binding site was amplified using mGC cDNA as a template and the following primers: $5^{\prime}-$ TATCTCGAGGAATTGAAGGCAGAGGCTTG-3' and 5'-TCGTCTAGATGACTCATCTCATGTGCCGTA-3'. To create the pmirGLO-Luc-Nurr1 3'-UTR WT vector, the resulting PCR fragment was cloned into the pmirGLO dual-luciferase miRNA target expression vector (Promega) using the XhoI and XbaI restriction sites (Promega). The mutant sequence contained two mutations in the 'seed sequence' of the miR-132 binding site, which is indicated in Fig. 5a. We designed primers (5'-CAGCTTTTGGATGTTTCCAGAG-3' and 5'-CACTCTGGAAACATCCAAAAGC-3') to create a pmirGLO-Luc-Nurr1 3'-UTR MU vector via overlap extension PCR. A luciferase reporter gene plasmid containing NGFI-B response elements upstream of the reporter (NBRE-Luc) was constructed according to previously described methods [26] using the pGL3-Basic vector (Promega), which was a generous gift from Sun Jianxin at Thomas Jefferson University, Philadelphia, USA. The sequences of all recombinant plasmids were confirmed by DNA sequencing.

\section{Transient transfection}

Chemically synthesized single-stranded RNAs that mimic mature endogenous miR-132 [GeneBank: NR_029546.1] after transfection into cells were used as miR-132 mimics, and mimics NC were used as negative controls. Chemically modified antisense RNA oligonucleotides optimized to specifically target miRNA molecules in cells were used as miRNA inhibitors, and inhibitors NC were used as negative controls. Nurr1-specific siRNA oligonucleotides (sense: 5'-CCACCUUGCUUGUACCAAAdTdT-3'; antisense: 3'-dTdT GGUGGAACGAACAUGGUUU-5') were used to knock down endogenous Nurr1, and siNC oligonucleotides were used as negative controls. These oligonucleotides were purchased from Ribobio (Guangzhou, China). Primary mGCs were transfected with either oligonucleotides or plasmids using Lipofectamine 2000 (Invitrogen) according to the manufacturer's protocol. For each transfection, a final oligonucleotide concentration of $100 \mathrm{nM}$ was used.

\section{Western blot analysis}

Total protein was isolated from mGCs that were harvested $48 \mathrm{~h}$ after treatment. The cells were rinsed twice with ice-cold PBS (pH 7.4) and lysed with whole lysis buffer $(50 \mathrm{mM}$ Tris- $\mathrm{HCl}, \mathrm{pH} 7.6 ; 150 \mathrm{mM} \mathrm{NaCl}$; and $1.0 \%$ NP-40) containing protease inhibitor cocktail 
(Sigma). The protein concentrations were measured using the Pierce BCA protein assay (Thermo). Equal amounts of total protein $(40 \mu \mathrm{g})$ were separated on a $10 \%$ SDS-polyacrylamide gel and transferred to a polyvinylidene fluoride membrane (Millipore, Billerica, MA, USA). Immunoblotting was performed using primary antibodies against Nurr1 (1:1000; R\&D Systems, Minneapolis MN, USA) and Nur77 (1:500; Santa Cruz, CA, USA). Glyceraldehyde-3-phosphate dehydrogenase (GAPDH) was selected as an internal control and was visualized using rabbit anti-GAPDH IgG (1:10000; Bioworld). Immunodetection was accomplished using a goat anti-rabbit IgG (1:5000; GenScript, Piscataway, NJ, USA) or a donkey anti-goat IgG (1:5000; Santa Cruz) secondary antibody and an enhanced chemiluminescence detection kit (Millipore) with the Clinx Chemiscope 3400 Mini Western Blot Imaging System (Clinx Science Instruments, Shanghai, China). Signals from the Western blot images were quantified by measuring the optical density of each band. The blot density of the control was set as $100 \%$. After normalization to the corresponding GAPDH band, the relative density values of other bands were calculated by dividing the optical density values by the control value. All experiments were repeated three times.

\section{RNA extraction and quantitative real-time PCR}

Total RNA was extracted from cultured cells using the TRIzol reagent (Invitrogen). cDNA was synthesized from $1 \mu \mathrm{g}$ of purified total RNA using the PrimeScript RT Reagent Kit with gDNA Eraser (Takara, Dalian, China) according to the manufacturer's instructions with either the random primers provided in the kit or specific reverse primers (miR-132: 5'-CTCAACTGGTGTCGTGG AGTCGGCAATTCAGTTGAGCGACCATG-3', U6: 5'AACGCTTCACGAATTTGCGT-3'). The specific primers used for real-time PCR analysis are listed in Table 1. Each $20 \mu \mathrm{L}$ real-time PCR reaction had the following components: $2 \mu \mathrm{L}$ of RT product (equivalent to $20 \mathrm{ng}$ of total RNA), $10 \mu \mathrm{L}$ of iQ SYBR Green Supermix (Bio-Rad Laboratories, Hercules, CA, USA), and $250 \mathrm{nM}$ forward and reverse primers. Real-time PCR for gene transcription was performed on a MyiQ Single Color Real-time PCR Detection System (Bio-Rad Laboratories). The cycle parameters for miRNAs were as follows: an initial $15 \mathrm{~min}$ incubation at $95{ }^{\circ} \mathrm{C}$, followed by 40 cycles of $95^{\circ} \mathrm{C}$ for $15 \mathrm{~s}$ and $60{ }^{\circ} \mathrm{C}$ for $1 \mathrm{~min}$. The cycle parameters for genes were as follows: an initial $3 \mathrm{~min}$ incubation at $95{ }^{\circ} \mathrm{C}$, followed by 40 cycles of $95{ }^{\circ} \mathrm{C}$ for $10 \mathrm{~s}, 60^{\circ} \mathrm{C}$ for $30 \mathrm{~s}$, and $72{ }^{\circ} \mathrm{C}$ for $30 \mathrm{~s}$. The data were analyzed using the $2^{-\Delta \Delta \mathrm{Ct}}$ method [27], and the obtained fold changes in miRNA or gene expression were normalized to U6 snRNA or 18S rRNA as endogenous controls, respectively. Each sample was analyzed in triplicate, and the experiments were repeated three times.

\section{Luciferase reporter assay}

mGCs with a confluency of $\sim 60 \%$ were transfected with luciferase reporter plasmids and miR-132 mimics/inhibitors or the corresponding negative controls. All cells were co-transfected with the Renilla luciferase reporter plasmid (pRL-RSV; Promega) as a control for transfection efficiency. Luciferase activity was assayed $48 \mathrm{~h}$ after transfection using the Dual-Luciferase Reporter Assay System (Promega), and the ratio of firefly luciferase to Renilla luciferase was measured using a Centro XS3 LB 960 Microplate Luminometer (Berthold Technologies, Bad Wildbad, Germany). At least three transfection assays were performed to obtain statistically significant data.

\section{Hormone assays}

For hormone assays, mGCs were cultured in 12-well plates in phenol red-free DMEM/F12 (Hyclone) supplemented with $2 \%$ C-FBS (HyClone) and $2 \mu \mathrm{M} 4$ androstene-3, 17-dione (Sigma). To determine the effects of 8-Br-cAMP on mGC function, mGCs were treated with medium alone or with $1 \mathrm{mM}$ 8-Br-cAMP (Sigma) for $24 \mathrm{~h}$ or $48 \mathrm{~h}$. To determine the effects of miR-132 on mGCs, the medium was changed $6 \mathrm{~h}$ after transfection with miR132 mimics/inhibitors or the corresponding negative controls, and the cells were cultured for an additional $48 \mathrm{~h}$. To determine the effect of Nurr1 on mGCs, siNurr1 was transfected into cells $24 \mathrm{~h}$ prior to the transfection of miR-132 mimics, and the cells were cultured for an additional $24 \mathrm{~h}$ or $48 \mathrm{~h}$. Culture medium was collected at the indicated time points, and the concentrations of $E_{2}$ and progesterone in the culture medium were determined using the Access Immunoassay System 2 (Beckman Coulter, Brea, CA, Germany), an automated

Table 1 Sequences of primers used for real-time PCR analysis

\begin{tabular}{lll}
\hline Gene & Forward primer $\left(5^{\prime} \rightarrow 3^{\prime}\right)$ & Reverse primer $\left(5^{\prime} \rightarrow 3^{\prime}\right)$ \\
\hline miR-132 & ACACTCCAGCTGGGTACAGTCTACAGCCA & GGTGTCGTGGAGTCGGCAATTCAGTTGAG \\
U6 snRNA & CTCGCTTCGGCAGCACA & AACGCTTCACGAATTTGCGT \\
Cyp19a1 & TGTGTTGACCCTCATGAGACA & CTTGACGGATCGTTCATACTTC \\
Nurr1 & TCCCTGTAAATGGGGCCATAC & AGGTCCTTCAATGAGATCCCTT \\
185 rRNA & GATCGAGCAGAGGAAGAC & AAGCGCATCTGGCAGCTA \\
\hline
\end{tabular}


random-access chemiluminescence-based assay. The intra- and interassay coefficients of variation were less than $10 \%$ and $15 \%$, respectively. Each assay was performed in triplicate, and the experiments were repeated at least three times.

\section{Statistical analysis}

Data were expressed as the mean $+/$ - SEM of at least three independent experiments. Student's $t$-test was performed for comparisons of the mean values of two groups; one-way ANOVA was used to determine differences among the mean values of more than two groups because the quantitative data followed a normal distribution. $P$ values less than 0.05 were considered statistically significant.

\section{Results}

miR-132 expression is responsive to 8-Br-cAMP stimulation As shown in Fig. 1a, we successfully established monolayers of mGCs from the ovaries of 21-day-old immature mice. Most of the cells in the cultures were mGCs, which were characterized by positive FSHR staining. FSHR expression also showed that our in vitro cultured primary mGCs can maintain an estrogenic stage and can be used for further study of $E_{2}$ synthesis. To test the specificity of anti-FSHR antibody, we did immunohistochemistry to show that our primary anti-FSHR antibody exclusively detected FSHR (stained brown) mainly in the mGCs at various stages of follicular development. Non-specific staining was not detected with PBS (Fig. 1b). The secretion of progesterone and $E_{2}$ by cultured mGCs was significantly increased (4.6- and 1.3-fold, respectively) after exposure to 8-Br-cAMP, a stable cell-permeable analog of cAMP, for $24 \mathrm{~h}$ (Fig. 1c, d). The stimulatory effect of 8-Br-cAMP on $\mathrm{E}_{2}$ reached a greater extent $(1.5$-fold) after continuous treatment with 8-Br-cAMP for $48 \mathrm{~h}$ (Fig. 1d). cAMP is the crucial second messenger that is downstream of FSH in the FSH-mediated ovarian GC differentiation pathway. At least four cAMP-response element (CRE) sites are involved in miR-132 transcription in mice [28]. Previous studies have demonstrated that miR-132 levels are elevated in periovulatory mGCs and upregulated by hCG/ LH, cAMP and FSH [19-21]. To determine whether miR132 is induced by the cAMP signal transduction pathway, primary mGCs were exposed to 8-Br-cAMP for 0 to $48 \mathrm{~h}$. During this period, miR-132 expression was continuously elevated, peaking at $12 \mathrm{~h}(\sim 5$-fold increased $)$ and dropped to basal level after $24 \mathrm{~h}$ (Fig. 1e). The observed pattern of miR-132 upregulation is consistent with reported increases in steroid hormone release from mGCs, suggesting that miR-132 is involved in cAMP-mediated pathways, such as those that are important for the differentiation of GCs.
miR-132 enhances the synthesis of $E_{2}$ in mGCs

Next, we assessed whether miR-132 has an effect on steroidogenesis in mGCs. To elevate miR-132 levels in mGCs, we transiently transfected mGCs with miR-132 mimics (i.e., chemically modified oligonucleotides) and confirmed the increased miR-132 levels using qRT-PCR (Fig. 2a). The progesterone levels changed only slightly (Fig. 2b). However, the $\mathrm{E}_{2}$ levels significantly increased after miR-132 overexpression. This increase was dose dependent; $35 \%$ and $72 \%$ increases in the $\mathrm{E}_{2}$ levels were observed when cells were transfected with $50 \mathrm{nM}$ and $100 \mathrm{nM}$ miR-132 mimics, respectively (Fig. 2c). In addition, we studied miR-132-related loss-of-function by knocking down endogenous miR-132 via the transient transfection of miR-132 inhibitors into mGCs (Fig. 3a). The results demonstrated that the synthesis of $E_{2}$ was suppressed by miR-132 knockdown and downregulation of miR-132 prevented a cAMP-mediated increase of $E_{2}$ in mGCs (Fig. 3b). The above findings suggest that miR-132 can serve as a stimulator for $\mathrm{E}_{2}$ synthesis in GCs. The detection of estrogen synthesis-related genes using real-time PCR revealed that a 1.6-fold increase in the expression of Cyp19a1 $(P<0.01)$, the P450 aromatase gene required for $\mathrm{E}_{2}$ synthesis, was induced by miR-132 mimics (Fig. 4a). However, the expression of Cyp11a1, a key gene for progesterone synthesis, was not influenced by the overexpression of miR-132 (Fig. 4a). Significant effects on Cyp11a1 expression were not observed after the knockdown of miR-132 in the presence or absence of 8-Br-cAMP treatment (Fig. 4b). In contrast, the observed effect of miR-132 inhibitors on Cyp19a1 levels was similar to the suppression in $\mathrm{E}_{2}$ levels (Fig. $3 \mathrm{~b}$ ), and this effect became notable after 8-Br-cAMP treatment in mGCs (Fig. 4c). These findings suggest that miR-132 promotes $\mathrm{E}_{2}$ synthesis via the transcriptional regulation of aromatase but has little effect on progesterone synthesis due to its failure to regulate the transcription of Cyp11a1.

\section{miR-132 promotes Cyp19a1 expression by downregulating Nurr1}

miRNAs suppress translation by targeting the 3'-UTRs of mRNAs. We hypothesized that miR-132 promotes Cyp19a1 expression by suppressing a Cyp19a1 inhibitor. A bioinformatics screen using TargetScan (http:// www.targetscan.org/vert_61/) revealed that the orphan nuclear receptor Nurr1, which suppresses aromatase expression via its PII promoter in KGN cells [29], is a putative target gene of miR-132. We constructed the luciferase reporter plasmid pmirGLO-Luc-Nurr1 3'-UTR WT, which contained the 3'-UTR of mouse Nurr 1 and the putative binding site for the 'seed sequence' of miR-132 (Fig. 5a), and co-transfected it into mGCs with either miR-132 mimics/inhibitors or the corresponding negative controls. Compared to controls, the overexpression of 


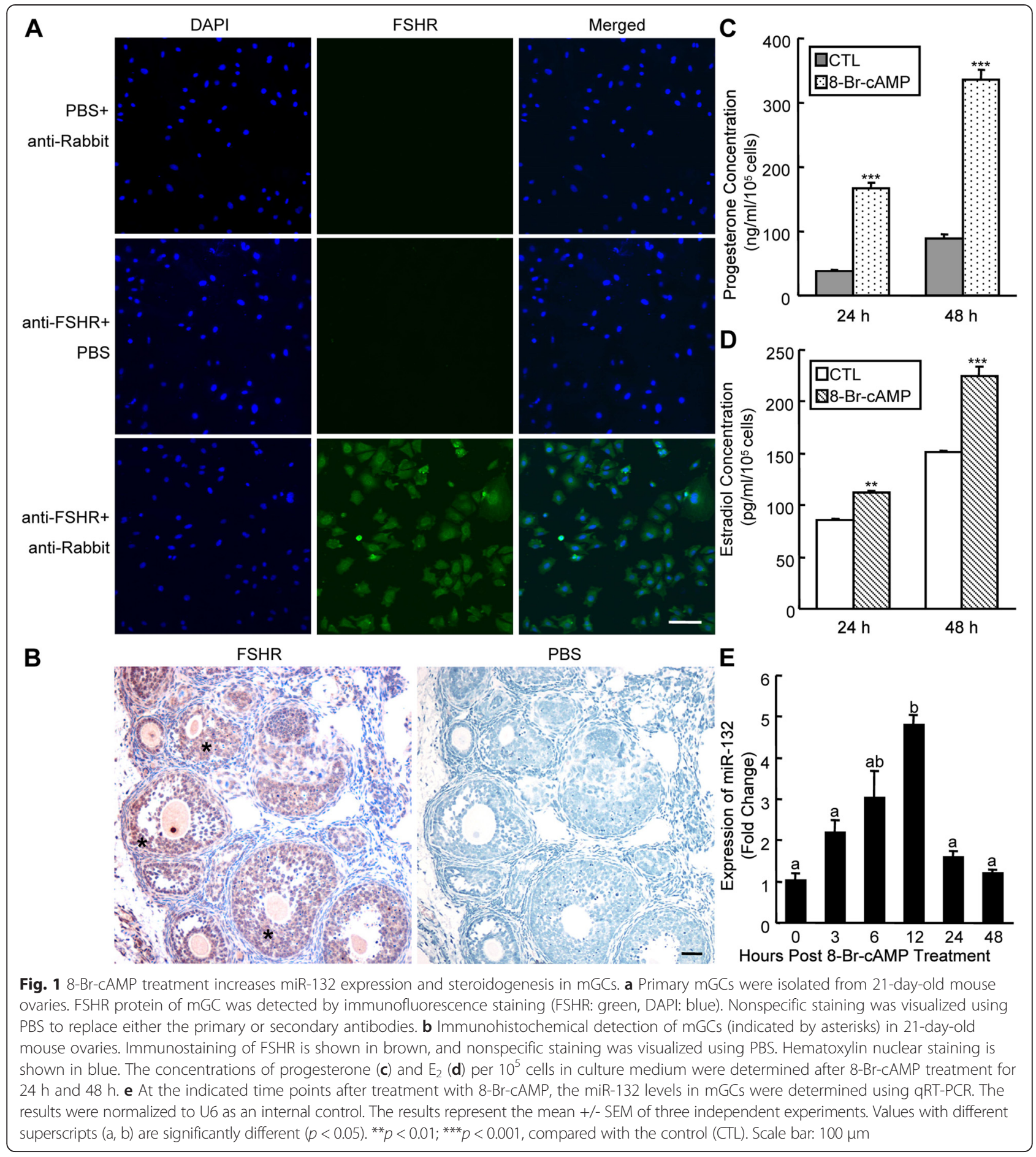

miR-132 significantly decreased luciferase activity, and the knockdown of miR-132 significantly increased luciferase activity in transfected mGCs (Fig. 5b), indicating that Nurr1 is a direct target of miR-132. In addition, we constructed the pmirGLO-Luc-Nurr1 3'-UTR MU plasmid, which had two mutations in the 'seed sequence' of the miR-132 binding site, as indicated in Fig. 5a. miR-132 failed to affect the luciferase activity of the mutagenized
Nurr1 3'-UTR plasmid (Fig. 5c). These results indicated that Nurr1 is a direct target gene of miR-132. The NR4A orphan nuclear factors bind as monomers to the NBRE motif (5'-AAAAGGTCA-3') and function in ligandindependent transcription activation. We utilized the NBRE-Luc luciferase plasmid, which contains tandem copies of the response elements for NR4A and drives a luciferase reporter gene, as a response reporter to reflect 


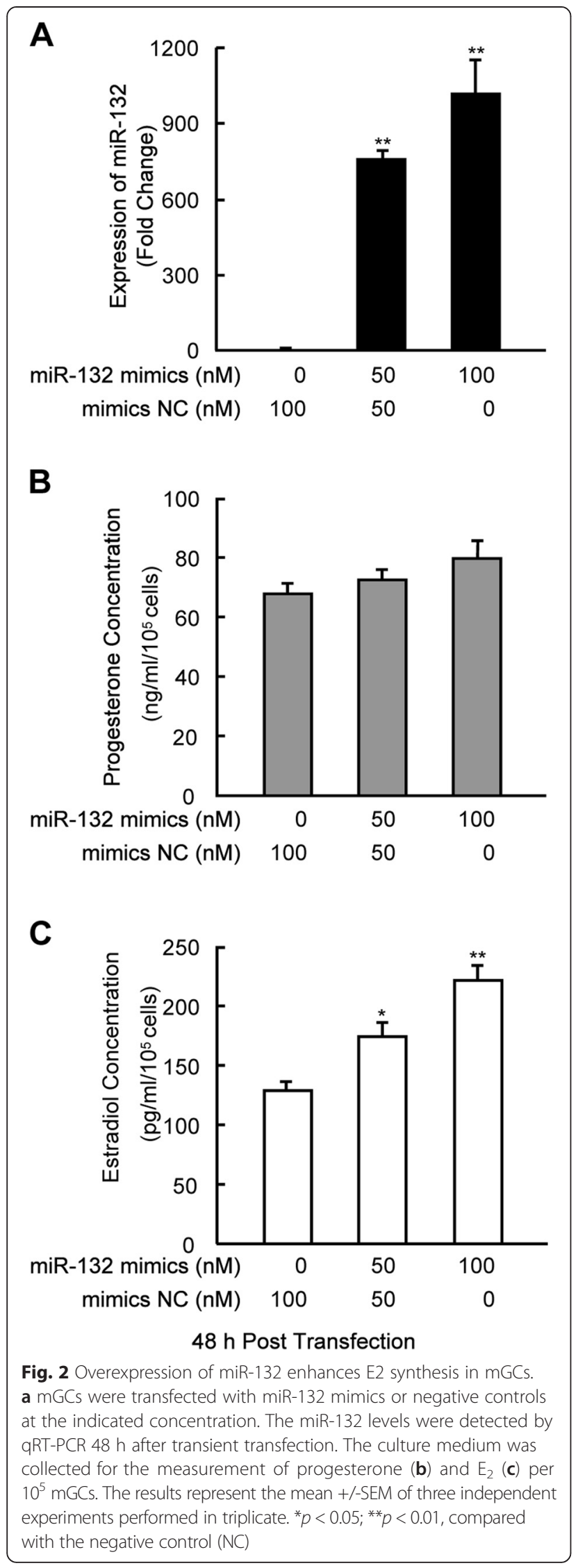

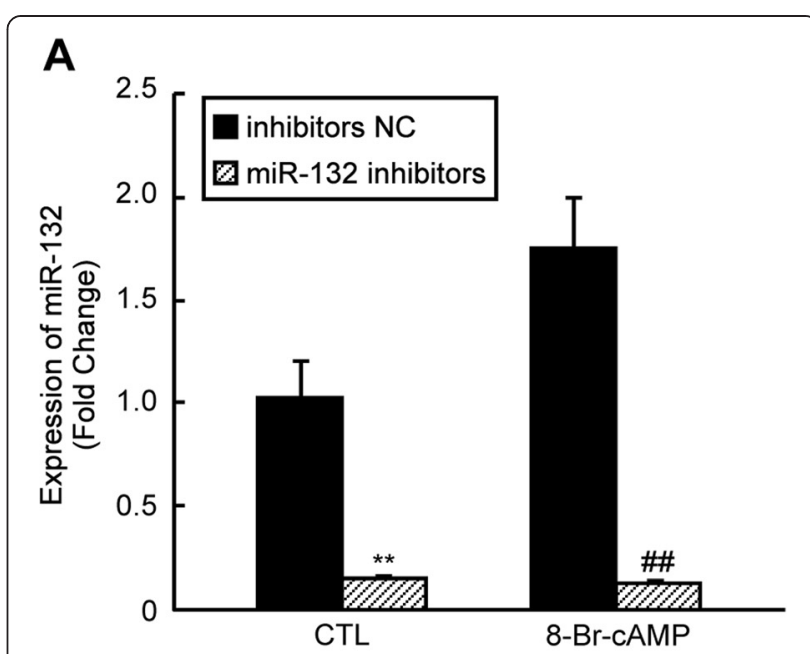

B

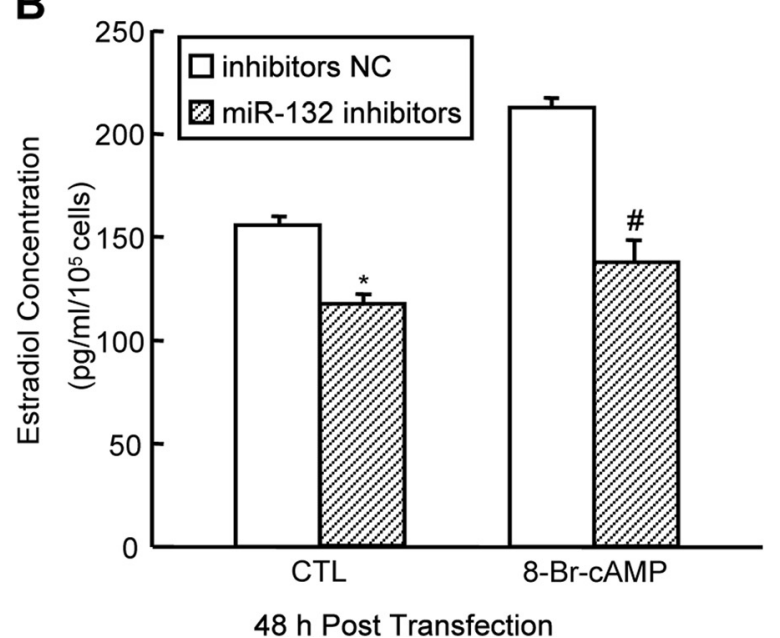

Fig. 3 Downregulation of endogenous miR-132 inhibits E2 synthesis in mGCs. a mGCs were transfected with $100 \mathrm{nM}$ miR-132 inhibitors or negative controls. Six hours after transfection, mGCs were cultured in the absence or presence of 8-Br-cAMP for another $48 \mathrm{~h}$. The inhibition of endogenous miR-132 by specific inhibitors was validated by qRT-PCR. $\mathbf{b}$ The culture medium was collected for the measurement of $E_{2}$ levels after endogenous miR-132 had been knocked down. The results represent the mean $+/$ - SEM of three independent experiments performed in triplicate. ${ }^{*}, \# p<0.05 ;{ }^{*} p<0.01$; \#\# $p<0.005$, compared with the negative control (NC)

transcriptional activity of the NR4A family. The luciferase assay demonstrated that miR-132 suppressed the luciferase activity of the NBRE-Luc reporter gene. Our findings were further supported by the observation that the induction of the NR4A family was observed after the inhibition of endogenous miR-132 via miR-132 inhibitors (Fig. 5d). Analysis of Nurr1 levels via Western blot analysis demonstrated that Nurr1 protein expression was significantly lower in mGCs that were transfected with miR-132 mimics than in mGCs that were transfected with mimics NC. In contrast, compared to treatment with inhibitors $\mathrm{NC}$, knockdown of miR-132 using miR-132 inhibitors led 


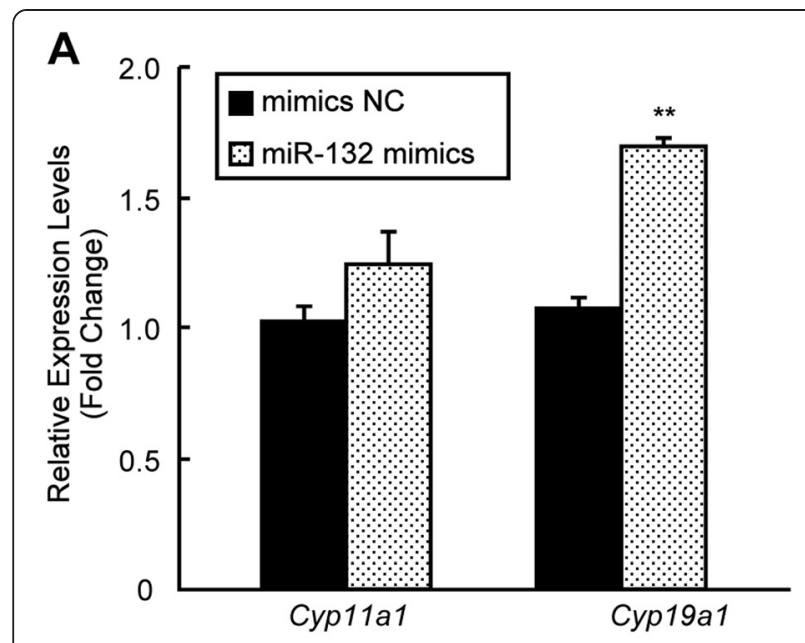

B

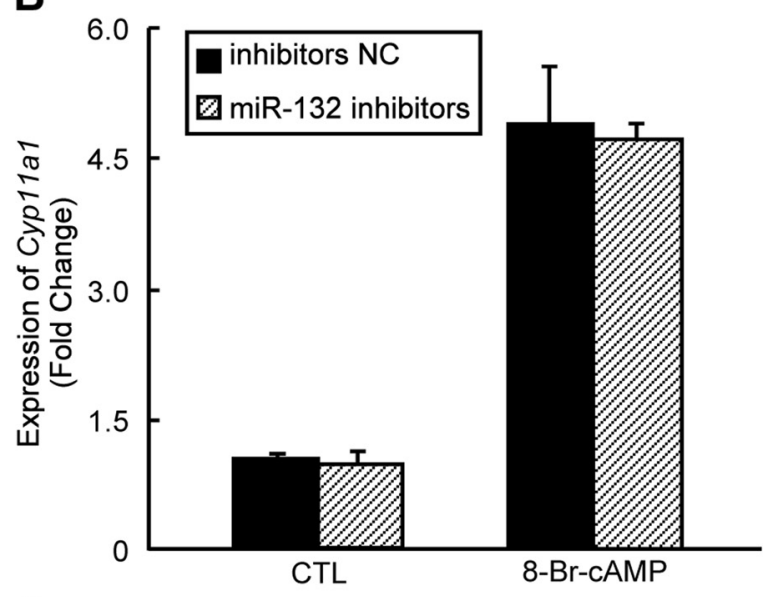

C

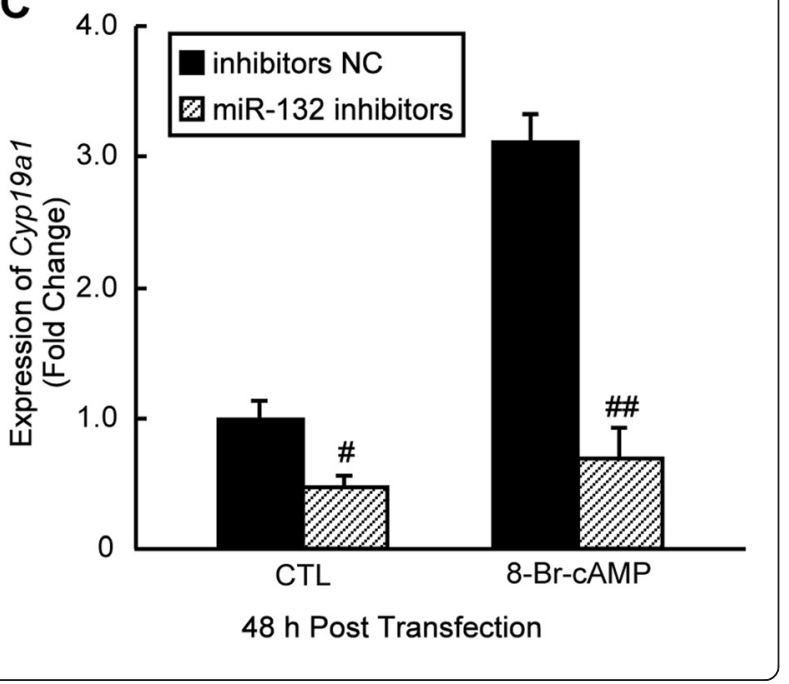

Fig. 4 Effects of miR-132 on Cyp11a1 and Cyp19a1 in mGCs. To examine the effect of miR-132 on Cyp11a1 and Cyp191a1 transcription, mGCs were transfected with $100 \mathrm{nM}$ of miR-132 mimics (a) or $100 \mathrm{nM}$ of miR-132 inhibitors in the absence (b) or presence of 8-Br-cAMP (c) for $48 \mathrm{~h}$ as indicated, or the corresponding negative controls. Total RNA was extracted from mGCs $48 \mathrm{~h}$ after transfection, and the mRNA expression levels of Cyp11a1 and Cyp19a1 were measured by real-time PCR. The results represent the mean +/-SEM of three independent experiments. \# $p<0.05$; ${ }^{*} p<0.01$; \#\# $p<0.005$, compared with the negative control (NC)

to increased Nurr1 protein expression in mGCs (Fig. 5e). These results were consistent with the findings of a previous study of the differentiation of dopamine neurons [30]. Significant changes in Nurr1 mRNA levels were not observed after either the overexpression or knockdown of miR-132 (Fig. 5f). In the 3'-UTR of Nur77, which has also been identified as a repressor of aromatase in NR4A family members, no putative binding site for miR-132 was found via bioinformatics screening. The expression level of the Nur77 protein was not influenced by miR-132 (Fig. 5e) in our study. In summary, miR-132 post-transcriptionally inhibits the translation of Nurr1 and weakens its repressive effect on Cyp19a1 transcription. This miR-132-mediated reduction of Nurr1 repression leads to Cyp19a1 upregulation and increased $\mathrm{E}_{2}$ synthesis.

\section{Knockdown of Nurr1 partially attenuates the effects of miR-132 and re-expression of Nurr1 abrogates the stimulatory effect of miR-132 on $E_{2}$ synthesis}

The knockdown of Nurr1 via RNA interference was validated using both Western blot analysis and real-time PCR (Fig. 6a, b). The $E_{2}$ synthesis was primarily elevated after Nurr1 knockdown via siNurr1 transfection compared to siNC (Fig. 6c, the left panels), which was similar to the previously observed effect of the downregulation of Nurr1 by miR-132. Followed by transfection of miR132 mimics for $48 \mathrm{~h}$, miR-132 significantly promoted $\mathrm{E}_{2}$ synthesis as expected in siNC group, while miR-132 failed to further contribute to the elevation of the $E_{2}$ synthesis in siNurr1 group (Fig. 6c, the right panels). In addition, the Cyp19a1 mRNA levels showed similar changes (Fig. 6d). After transfecting mGCs with FlagNurr1, which upregulated Nurr1 protein levels independent of miR-132 repression due to the absence of the 3'-UTR binding sequence for miR-132 in the pFLAGCMV-2 expression plasmid, the stimulatory effect of miR-132 on $E_{2}$ synthesis was largely abrogated (Fig. 6e). The $E_{2}$ levels dropped to basal levels. These results suggest that Nurr1 plays an important role in miR-132induced $\mathrm{E}_{2}$ synthesis.

\section{Discussion}

Both FSH and LH promote intracellular cAMP in GCs via binding to their receptors [23]. We isolated naïve GCs 

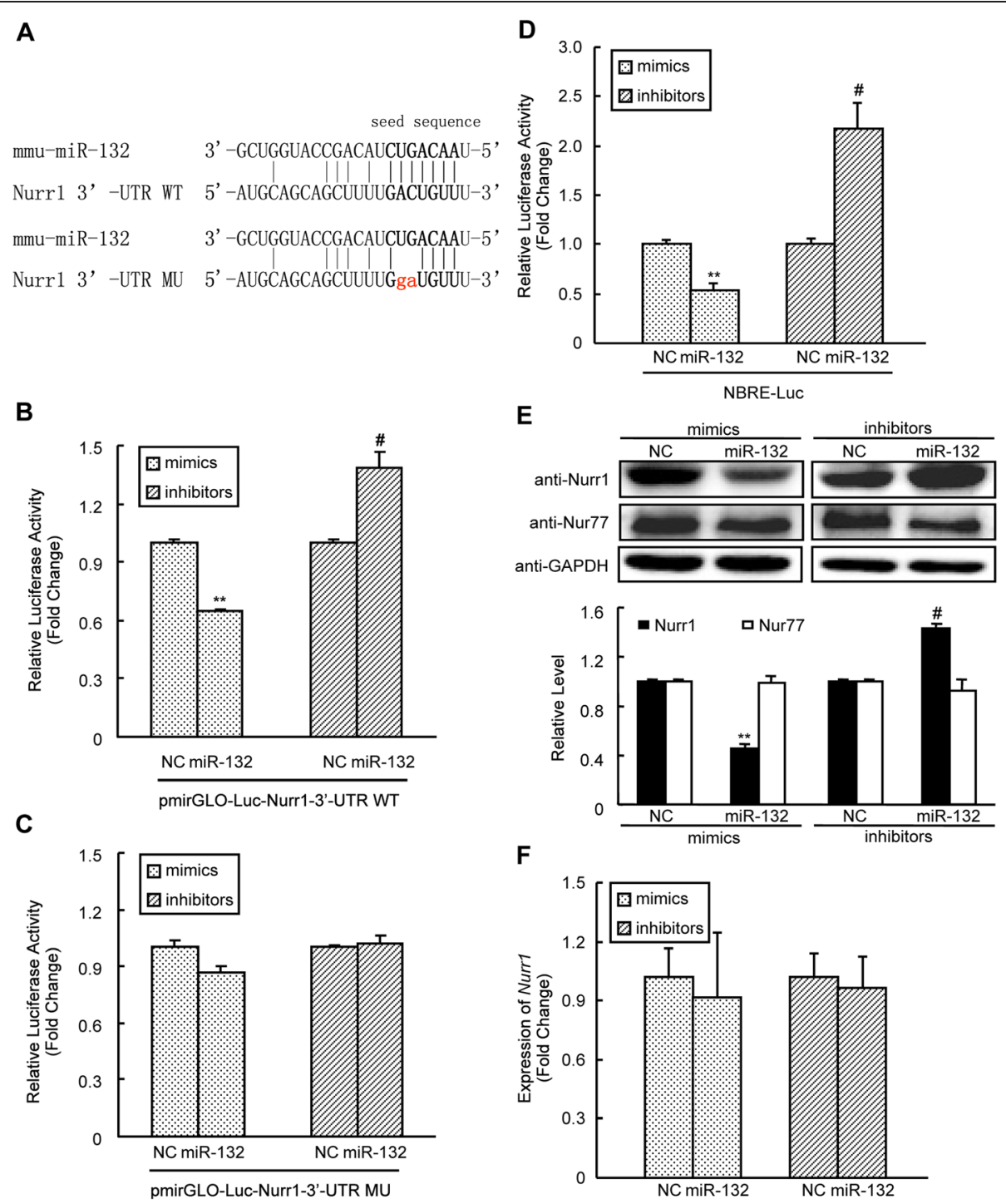

$\mathbf{F}$

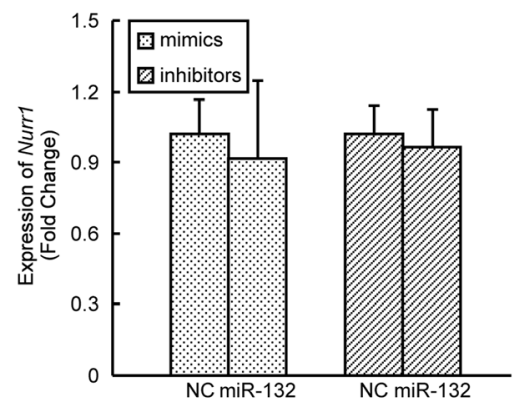

Fig. 5 miR-132 suppresses Nurr1 expression post-transcriptionally. a The putative site in the Nurr1 3'-UTR that contains the 'seed sequence' for miR-132 binding. The $g$ and a shown in red indicate the miR-132 binding site in the mutant form of Nurr1. mGCs were transfected with 100 nM miR-132 mimics, miR-132 inhibitors or the corresponding negative controls. Cells were co-transfected with the wild-type (WT) Nurr1 3'-UTR luciferase reporter plasmid (b), the mutant (MU) Nurr1 3'-UTR luciferase reporter plasmid (c) or the NBRE-luciferase reporter plasmid (d). After 48 h, luciferase assays were performed, and the results were normalized using constitutive Renilla luciferase. e Western blot analysis of Nurr1 and Nur77 protein expression in mGCs 48 h after transfection with $100 \mathrm{nM}$ of miR-132 mimics, miR-132 inhibitors or the corresponding negative controls. The upper panels depict representative Western blots, and the lower panels present the statistical summary of the densitometric analysis from three independent experiments, indicating expression levels relative to negative controls after normalization to GAPDH. $\mathbf{f}$ Real-time PCR analysis of Nurr1 mRNA levels in mGCs $48 \mathrm{~h}$ after transfection. The results represent the mean +/-SEM of three independent experiments performed in triplicate. ${ }^{\star *} p<0.01 ; \# p<0.05$, compared with the negative control (NC)

from immature mice and used 8-Br-cAMP to mimic the secondary messenger downstream of the FSH pathway. Our in vitro analysis of miR-132 expression in cultured mGCs treated with 8-Br-cAMP demonstrated that miR132 levels were significantly upregulated and peaked at $12 \mathrm{~h}$ (Fig. 1e). The induction of miR-132 was also observed in FSH or cAMP -treated rat GCs [19, 20]. The treatment of mouse ovaries with an ovulatory dose of LH/
hCG revealed that miR-132 was highly upregulated in periovulatory mGCs [21]. The results of previous studies are consistent with our findings, which demonstrated that miR-132 was induced by hormonal stimulation and activation of the cAMP pathway in GCs. A previous study demonstrated that miR-132 is regulated by CREB via CRE motifs upstream of miR-132 [31]; this finding explains the observed upregulation of miR-132 by cAMP activation in 


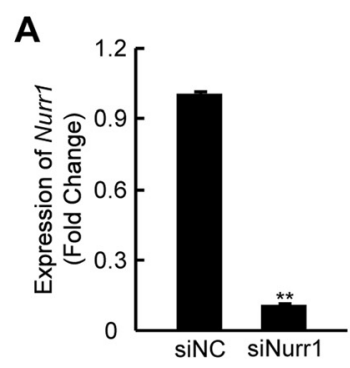

B
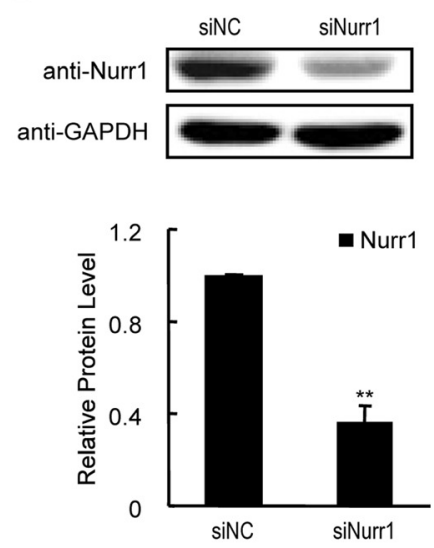

C

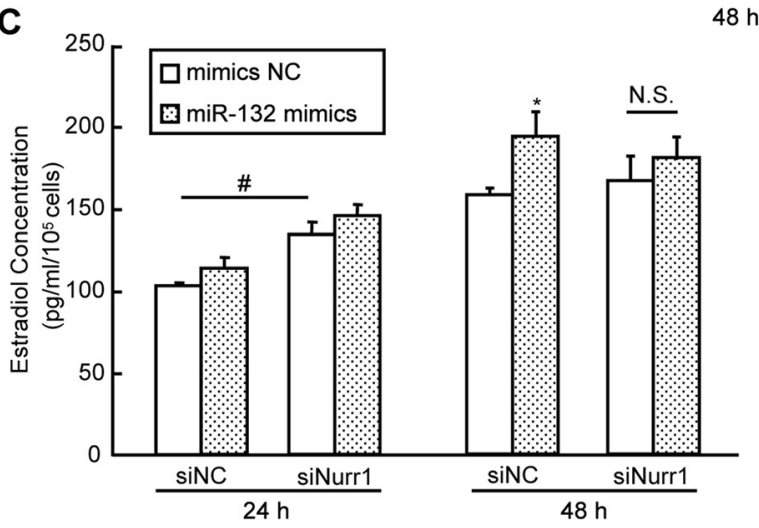

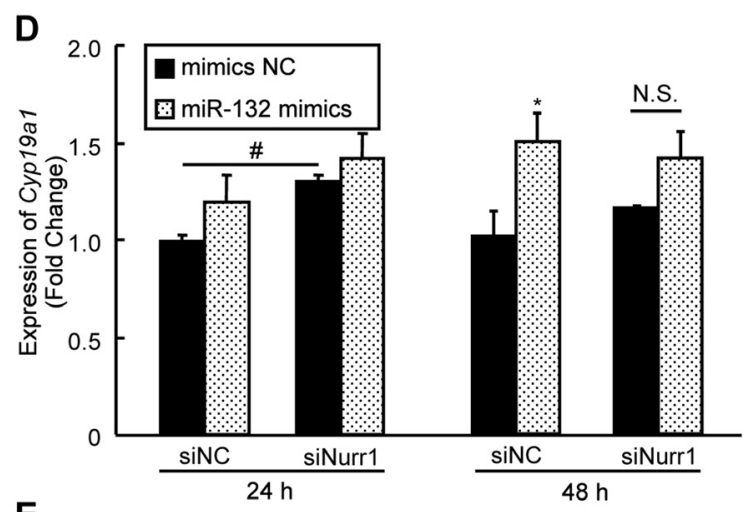

$\mathbf{E}$

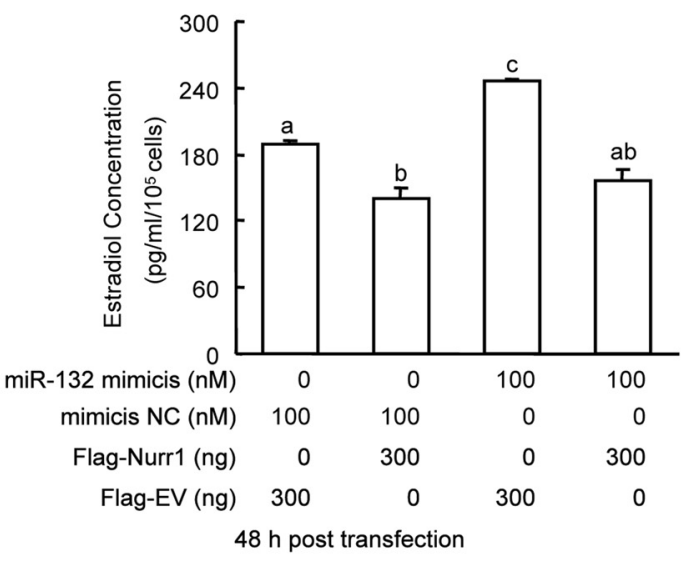

Fig. 6 Knockdown of Nurr1 partially attenuates the effects of miR-132, and re-expression of Nurr1 abrogates the stimulatory effect of miR-132 on E2 synthesis. a mGCs were transfected with 100 nM siRNA targeting Nurr1 or negative control siRNA. Real-time PCR detection of Nurr1 mRNA levels was performed $48 \mathrm{~h}$ after transfection of mGCs. b After knockdown, Nurr1 protein levels were analyzed by Western blot. c siRNA was transfected $24 \mathrm{~h}$ before transfection of miR-132 mimics or negative controls, followed by continuous culture for an additional 48 h. The culture medium was collected for the measurement of $\mathrm{E}_{2}$ levels $24 \mathrm{~h}$ and $48 \mathrm{~h}$ after transfection. $\mathbf{d}$ Total RNA was extracted from mGCs and Cyp19a1 mRNA levels were measured using real-time PCR. e Culture medium was collected for the measurement of $E_{2}$ levels $48 \mathrm{~h}$ after transfecting mGCs with the Flag-Nurr1 or the Flag-empty vector (EV) plasmids and miRNA mimics, as indicated. The results represent the mean $+/$-SEM of three independent experiments performed in triplicate. Values with different superscripts $(a, b, c)$ are significantly different $(p<0.05)$. ${ }^{*} p<0.05$; ${ }^{* *} p<0.01$; N.S. no significant difference, compared with the negative control (NC)

GCs. These findings also suggest that miR-132 mediates functions of the cAMP pathway during the differentiation process of GCs.

There is increasing interest in identifying the functions of miRNAs in GCs. miR-21, which in addition to miR132 and miR-212, is an LH-induced miRNA, blocks apoptosis in mGCs [32]. The TGF- $\beta /$ Smads signaling pathway plays critical roles in early follicle development, GC proliferation and differentiation. Our previous study demonstrated that miR-145 and miR-181a suppress the proliferation of mGCs by targeting Acvr1b and Acvr2a, respectively [25, 33]. This pathway also regulates the expression of many miRNAs, including miR-224 and miR-383 [15, 16]. Elevated miR-224 can enhance TGF- 
$\beta 1$-induced mGC proliferation by targeting Smad4 and ovarian $E_{2}$ release [15], while the downregulation of miR-383 promotes steroidogenesis by targeting RBMS1 and can be transactivated by SF-1 through direct binding to the promoter of the miR-383 host gene SGCZ [16]. In porcine GCs, miR-378 is spatiotemporally expressed and shows an inverse expression pattern to that of aromatase. Aromatase expression and subsequent $\mathrm{E}_{2}$ production by $\mathrm{GCs}$ are directly post-transcriptionally downregulated by miR-378 [19]. In KGN cells, overexpression of miR-132 increased $E_{2}$ levels [22], which is consistent with our findings in mGCs. However, a study in equine follicle development found that miR-132 was increased in granulose cells from luteinizing follicles with higher progesterone and lower estradiol concentration in the follicular fluid [34]. In preovulatory mGCs, knockdown of miR-132 failed to affect estradiol or progesterone after cAMP treatment [21]. In a genome-scale screen of steroid hormone release influenced by miRNAs in human primary ovarian GCs, 51 miRNAs were found to suppress $\mathrm{E}_{2}$ release, whereas none of the miRNAs (including miR132) studied were found to have a stimulatory effect on the $E_{2}$ level [14]. This discrepancy could be attributed to differences between species and cell models. miR-132 may exhibit diverse functions at specific stages of GCs development. Therefore, we utilized a lower plating density to retain an estrogenic phenotype of GCs. Our data suggest that $\mathrm{E}_{2}$ production and the Cyp19a1 mRNA levels in mGCs are elevated by miR-132 directly. Our loss-offunction study also demonstrated that the knockdown of miR-132 could downregulate the expression of Cyp19a1. Consequently, the increased levels of miR-132 after 8-BrcAMP treatment could contribute to the extended suppressive effect of miR-132 inhibitors on Cyp19a1. Taken together, miR-132 was induced by cAMP and likely mediated the FSH pathway in the primary cultured mGCs that we studied because of its stimulatory effect on $E_{2}$ synthesis. To better understand the functions of miR-132 in GCs of terminal differentiation (e.g. apoptosis), further studies are needed.

In addition, our research elucidated some of the molecular mechanisms that underlie the stimulatory effect of miR-132 on $E_{2}$ synthesis. We hypothesized that miR-132 stimulates $E_{2}$ synthesis via translational regulation of an orphan nuclear receptor-Nurr1. Orphan nuclear receptors in the ovary, such as SF-1, which is also known as NR5A1 [6, 35], are emerging as important ovarian factors that regulate female reproduction. The orphan nuclear receptor Nurr1 belongs to the nuclear receptor subfamily 4A (NR4A) subgroup along with Nur77 and Nor1 [29]. The genes encoding these transcription factors are classified as immediate early response genes because their expression is rapidly induced by a variety of physiological stimuli, including fatty acids, prostaglandins, growth factors, calcium, cytokines and peptide hormones (e.g., FSH) [36]. NUR77 is a novel transcription factor that contributes to the regulation of prolactin gene expression in human endometrial stromal cells and regulates androgen receptor gene expression in ovarian GCs [37, 38]. Both NUR77 and NURR1 suppress the transcription of aromatase and modulate its expression in the KGN human granulosa-like tumor cell line [29]. In a recent study of embryonic stem cell differentiation, miR-132 was demonstrated to directly regulate the expression of Nurr1, which is an important transcription factor in dopamine neuron development and differentiation [29]. Our study demonstrates that miR-132 suppressed Nurr1 expression by targeting its 3 '-UTR (Fig. 5b). Interestingly, the Nurr1 protein levels in mGCs were dramatically decreased by the overexpression of miR-132 (Fig. 5e), whereas the Nurr1 mRNA levels were only slightly changed (Fig. 5f). This finding indicates that in mGCs, miR-132 induces Nurr1 translation inhibition but not mRNA degradation by binding to the 3'-UTR of Nurr1. It has been suggested that the promoter-proximal region of the aromatase PII promoter, which contains the binding sites for SF-1 and a CLS, also mediates the transcriptional repression of NURR1 and NUR77 [29]. However, this protein-DNA interaction might be too transient or too weak to be detected by the gel shift assay used in the previous study. The underlying mechanism by which NR4A mediates the transcriptional repression of Cyp19a1 remains to be elucidated. In contrast to the previously reported transient peaks in NR4A expression, the cAMP-mediated induction of miR-132 resulted in a delayed elevation pattern [29]. Conceivably, miR-132 expression could contribute to the decline of Nurr1 and the subsequent upregulation of Cyp19a1.

A previous study demonstrated that in neurons, miR132 is regulated by multiple factors, such as BDNF [39], and is required for both neuronal morphogenesis and long-term synapse activation [28]. Some targets of miR132, including p250GAP [40] and MeCP2 [41], have been identified. Interest in the involvement of miR-132 in endocrine biology has emerged recently. miRNA profiling in L $\beta \mathrm{T} 2$ cells exposed to gonadotropin-releasing hormone revealed the significant induction of miR-132, which subsequently regulated cellular motility [42]. Our study suggests that miR-132 may exert differential effects on reproductive endocrine regulation (e.g., the promotion of estrogen synthesis). In light of the important roles of both miR-132 and estrogen in brain function, it would be of interest to determine whether miR-132 influences local estrogen synthesis in the nervous system. In addition, the induction of miR-132 during Kaposi's sarcoma-associated herpes virus infection represses the expression of $\mathrm{p} 300$, a co-activator of CREB, which acts 
as part of a negative feedback loop that leads to the inhibition of miR-132 expression and the restoration of p300 expression [43]. This regulatory network may contribute to the observed decline in miR-132 levels after peak expression is reached during cAMP treatment. The precise regulatory role of $\mathrm{miR}-132$ and its functions in GCs remain to be elucidated. In addition, further in vivo studies, such as a study using floxed miR-212/132 mice [44] to specifically ablate miR-132 in GCs, could improve our understanding of the effect of miR-132 on $E_{2}$ synthesis. A recent study in polycystic ovary syndrome patients found that the expression levels of miRNA-132 in follicular fluid were significantly lower in patients than in controls [22]. The dysfunctions of miR-132 in the development of polycystic ovary syndrome and premature ovarian failure are to be elucidated in future studies.

\section{Conclusions}

In summary, our study demonstrated that cAMP induces the expression of miR-132 in mGCs; $E_{2}$ synthesis is subsequently induced by miR-132 via the upregulation of Cyp19a1. miR-132 induces Cyp19a1 by directly suppressing the expression of Nurr1. The observed effects of miR-132 on physiological processes in GCs may be useful for regulating reproduction and treating steroidrelated disorders.

\section{Abbreviations}

3'-UTR: 3'-untranslated region; CAMP: Cyclic adenosine monophosphate;

$E_{2}$ : Estradiol; GCs: Granulosa cells; miR-132: microRNA-132.

\section{Competing interests}

The authors declare that they have no competing interests.

\section{Authors' contributions}

SW, HS, GY and YH designed the study and prepared the manuscript. SW, QZ, YJ and TF performed in vitro primary GCs culture, immunofluorescence staining and immunohistochemistry. SW and YJ constructed the vectors. SW performed Western blot, and quantitative real-time PCR, and data analysis. IC assisted in data analysis and helped prepare the manuscript. All authors read and approved the final manuscript.

\section{Acknowledgements}

This work was supported by the National Natural Science Foundation of China (No. 81370724, No. 81370683, No. 81070492 and No. 81170570), a Special Grant for Principal Investigators from the Health Department of Jiangsu Province (No. XK201102, No. LJ201102, and No. RC2011005), a program of six top talents of Jiangsu Province, PR China (No. 2012-WSN-005). The authors thank Brianna M. Lutz for assistance with manuscript editing (New Jersey Medical School, Rutgers University).

\section{Author details}

${ }^{1}$ Reproductive Medicine Center, Department of Obstetrics and Gynecology, Nanjing Drum Tower Hospital, Nanjing University Medical School, Nanjing, China. ${ }^{2}$ New York-Presbyterian/Weill Cornell Medical Center, New York, NY, USA.

Received: 20 April 2015 Accepted: 13 August 2015

Published online: 19 August 2015

\section{References}

1. Drummond AE, Findlay JK. The role of estrogen in folliculogenesis. Mol Cell Endocrinol. 1999;151:57-64.
2. Huhtinen K, Stahle M, Perheentupa A, Poutanen M. Estrogen biosynthesis and signaling in endometriosis. Mol Cell Endocrinol. 2012;358:146-54.

3. Fanta M. Is polycystic ovary syndrome, a state of relative estrogen excess, a real risk factor for estrogen-dependant malignancies? Gynecol Endocrinol. 2013;29:145-7

4. Cordts EB, Christofolini DM, dos Santos AA, Bianco B, Barbosa CP. Genetic aspects of premature ovarian failure: a literature review. Arch Gynecol Obstet. 2011;283:635-43.

5. Senthilkumaran $B$, Yoshikuni M, Nagahama Y. A shift in steroidogenesis occurring in ovarian follicles prior to oocyte maturation. Mol Cell Endocrinol. 2004;215:11-8.

6. Ulloa-Aguirre A, Zarinan T, Pasapera AM, Casas-Gonzalez P, Dias JA. Multiple facets of follicle-stimulating hormone receptor function. Endocrine. 2007:32:251-63.

7. Silva JM, Hamel M, Sahmi M, Price CA. Control of oestradiol secretion and of cytochrome $\mathrm{P} 450$ aromatase messenger ribonucleic acid accumulation by FSH involves different intracellular pathways in oestrogenic bovine granulosa cells in vitro. Reproduction. 2006;132:909-17.

8. Michael MD, Kilgore MW, Morohashi K, Simpson ER. Ad4BP/SF-1 regulates cyclic AMP-induced transcription from the proximal promoter (PII) of the human aromatase P450 (CYP19) gene in the ovary. J Biol Chem. 1995;270:13561-6.

9. Bulun SE, Sebastian S, Takayama K, Suzuki T, Sasano H, Shozu M. The human CYP19 (aromatase P450) gene: update on physiologic roles and genomic organization of promoters. J Steroid Biochem Mol Biol. 2003;86:219-24.

10. Michael MD, Michael LF, Simpson ER. A CRE-like sequence that binds CREB and contributes to CAMP-dependent regulation of the proximal promoter of the human aromatase P450 (CYP19) gene. Mol Cell Endocrinol. 1997;134:147-56.

11. Ambros V. The functions of animal microRNAs. Nature. 2004;431:350-5.

12. Christenson LK. MicroRNA control of ovarian function. Anim Reprod. 2010;7:129-33

13. Baley J, Li J. MicroRNAs and ovarian function. J Ovarian Res. 2012;5:8.

14. Sirotkin AV, Ovcharenko D, Grossmann R, Laukova M, Mlyntek M. Identification of MicroRNAs controlling human ovarian cell steroidogenesis via a genome-scale screen. J Cell Physiol. 2009;219:415-20.

15. Yao G, Yin M, Lian J, Tian H, Liu L, Li X, et al. MicroRNA-224 is involved in transforming growth factor- $\beta$-mediated mouse granulosa cell proliferation and granulosa cell function by targeting Smad4. Mol Endocrinol. 2010;24:540-51.

16. Yin M, Lu M, Yao G, Tian H, Lian J, Liu L, et al. Transactivation of microRNA-383 by steroidogenic factor-1 promotes estradiol release from mouse ovarian granulosa cells by targeting RBMS1. Mol Endocrinol. 2012;26:1129-43.

17. Xu S, Linher-Melville K, Yang BB, Wu D, Li J. Micro-RNA378 (miR-378) regulates ovarian estradiol production by targeting aromatase. Endocrinology. 2011;152:3941-51.

18. Panda H, Chuang T-D, Luo X, Chegini N. Endometrial miR-181a and miR-98 expression is altered during transition from normal into cancerous state and target PGR, PGRMC1, CYP19A1, DDX3X, and TIMP3. J Clin Endocrinol Metab. 2012;97:E1316-26.

19. Hu Z, Shen WJ, Cortez Y, Tang X, Liu LF, Kraemer FB, et al. Hormonal regulation of microRNA expression in steroid producing cells of the ovary, testis and adrenal gland. PLoS One. 2013;8:e78040.

20. Yao N, Yang BQ, Liu Y, Tan XY, Lu CL, Yuan XH, et al. Follicle-stimulating hormone regulation of microRNA expression on progesterone production in cultured rat granulosa cells. Endocrine. 2010;38:158-66.

21. Fiedler SD, Carletti MZ, Hong X, Christenson LK. Hormonal regulation of MicroRNA expression in periovulatory mouse mural granulosa cells. Biol Reprod. 2008;79:1030-7.

22. Sang $Q$, Yao Z, Wang $H$, Feng $R$, Wang $H$, Zhao X, et al. Identification of microRNAs in human follicular fluid: characterization of microRNAs that govern steroidogenesis in vitro and are associated with polycystic ovary syndrome in vivo. J Clin Endocrinol Metab. 2013;98:3068-79.

23. Conti M. Specificity of the cyclic adenosine 3',5'-monophosphate signal in granulosa cell function. Biol Reprod. 2002;67:1653-61.

24. Portela VM, Zamberlam G, Price CA. Cell plating density alters the ratio of estrogenic to progestagenic enzyme gene expression in cultured granulosa cells. Fertil Steril. 2010;93:2050-5.

25. Yan G, Zhang L, Fang T, Zhang Q, Wu S, Jiang Y, et al. MicroRNA-145 suppresses mouse granulosa cell proliferation by targeting activin receptor IB. FEBS Lett. 2012;586:3263-70. 
26. Paulsen RE, Granas K, Johnsen H, Rolseth V, Sterri S. Three related brain nuclear receptors, NGFI-B, Nurr1, and NOR-1, as transcriptional activators. J Mol Neurosci. 1995;6:249-55.

27. Livak KJ, Schmittgen TD. Analysis of relative gene expression data using real-time quantitative PCR and the 2(-Delta Delta C(T)) method. Methods. 2001;25:402-8.

28. Wanet A, Tacheny A, Arnould T, Renard P. miR-212/132 expression and functions: within and beyond the neuronal compartment. Nucleic Acids Res. 2012;40:4742-53.

29. Wu YM, Ghosh S, Nishi Y, Yanase T, Nawata H, Hu Y. The orphan nuclear receptors NURR1 and NGFI-B modulate aromatase gene expression in ovarian granulosa cells: A possible mechanism for repression of aromatase expression upon luteinizing hormone surge. Endocrinology. 2005;146:237-46.

30. Yang $D$, Li T, Wang Y, Tang Y, Cui H, Tang Y, et al. miR-132 regulates the differentiation of dopamine neurons by directly targeting Nurr1 expression. J Cell Sci. 2012;125:1673-82.

31. Vo N, Klein ME, Varlamova O, Keller DM, Yamamoto T, Goodman RH, et al. A CAMP-response element binding protein-induced microRNA regulates neuronal morphogenesis. Proc Natl Acad Sci U S A. 2005;102:16426-31.

32. Carletti MZ, Fiedler SD, Christenson LK. MicroRNA 21 blocks apoptosis in mouse periovulatory granulosa cells. Biol Reprod. 2010;83:286-95.

33. Zhang $Q$, Sun $H$, Jiang $Y$, Ding L, Wu S, Fang T, et al. MicroRNA-181a suppresses mouse granulosa cell proliferation by targeting activin receptor IIA. PLoS One. 2013;8:e59667.

34. Schauer SN, Sontakke SD, Watson ED, Esteves CL, Donadeu FX. Involvement of miRNAs in equine follicle development. Reproduction. 2013;146:273-82.

35. Zhao H, Li Z, Cooney AJ, Lan ZJ. Orphan nuclear receptor function in the ovary. Front Biosci. 2007:12:3398-405.

36. Ding LJ, Yan GJ, Ge QY, Yu F, Zhao X, Diao ZY, et al. FSH acts on the proliferation of type A spermatogonia via Nur77 that increases GDNF expression in the Sertoli cells. FEBS Lett. 2011;585:2437-44.

37. Jiang $Y$, Hu Y, Zhao J, Zhen $X$, Yan G, Sun $H$. The orphan nuclear receptor Nur77 regulates decidual prolactin expression in human endometrial stromal cells. Biochem Biophys Res Commun. 2011:404:628-33.

38. Dai A, Yan G, He Q, Jiang Y, Zhang Q, Fang $T$, et al. Orphan nuclear receptor Nur77 regulates androgen receptor gene expression in mouse ovary. PLoS One. 2012;7:e39950

39. Remenyi J, Hunter CJ, Cole C, Ando H, Impey S, Monk CE, et al. Regulation of the miR-212/132 locus by MSK1 and CREB in response to neurotrophins. Biochem J. 2010;428:281-91.

40. Wayman GA, Davare M, Ando H, Fortin D, Varlamova O, Cheng HY, et al. An activity-regulated microRNA controls dendritic plasticity by down-regulating p250GAP. Proc Natl Acad Sci U S A. 2008;105:9093-8.

41. Klein ME, Lioy DT, Ma L, Impey S, Mandel G, Goodman RH. Homeostatic regulation of MeCP2 expression by a CREB-induced microRNA. Nat Neurosci. 2007;10:1513-4

42. Godoy J, Nishimura M, Webster NJG. Gonadotropin-releasing hormone induces miR-132 and miR-212 to regulate cellular morphology and migration in immortalized L $\beta T 2$ pituitary gonadotrope cells. Mol Endocrinol. 2011;25:810-20.

43. Lagos D, Pollara G, Henderson S, Gratrix F, Fabani M, Milne RS, et al. miR-132 regulates antiviral innate immunity through suppression of the p300 transcriptional co-activator. Nat Cell Biol. 2010;12:513-9.

44. Magill ST, Cambronne XA, Luikart BW, Lioy DT, Leighton BH, Westbrook GL, et al. microRNA-132 regulates dendritic growth and arborization of newborn neurons in the adult hippocampus. Proc Natl Acad Sci U S A. 2010;107:20382-7.

\section{Submit your next manuscript to BioMed Central and take full advantage of:}

- Convenient online submission

- Thorough peer review

- No space constraints or color figure charges

- Immediate publication on acceptance

- Inclusion in PubMed, CAS, Scopus and Google Scholar

- Research which is freely available for redistribution

Submit your manuscript at www.biomedcentral.com/submit 